\title{
Proton pump inhibitor intake negatively affects the osseointegration of dental implants: a retrospective study
}

\author{
Mehmet Ali Altay, Alper Sindel, Öznur Özalp, Nelli Yıldırımyan, Burak Kocabalkan \\ Department of Oral and Maxillofacial Surgery, School of Dentistry, Akdeniz University, Antalya, Turkey
}

\begin{abstract}
J Korean Assoc Oral Maxillofac Surg 2019;45:135-140)
Objectives: This study sought to investigate the association between the systemic intake of proton pump inhibitors (PPI) and the early failure of dental implants.

Materials and Methods: A retrospective cohort study involving 1,918 dental implants in 592 patients (69 implants in 24 PPI users and 1,849 implants in 568 nonusers, respectively) was conducted. The effect of PPI intake on the osseointegration of dental implants was evaluated using patientand implant-level models.

Results: Among 24 PPI users, two patients experienced implant failure, one of whom had three and the other of whom had one failed implant, respectively. Thus, the rate of failure for this population was $8.3 \%$. Separately, 11 nonusers each experienced one implant failure, and the failure rate for these patients was $1.9 \%$. Fisher's exact test revealed statistically significant differences between PPI users and nonusers at the implant level $(P=0.002)$ but failed to show any significance at the patient level $(P=0.094)$. The odds of implant failure were 4.60 times greater among PPI users versus nonusers. Dental implants that were placed in patients using PPIs were found to be 4.30 times more likely to fail prior to loading.

Conclusion: The findings of this study suggest that PPI intake may be associated with an increased risk of early dental implant failure.
\end{abstract}

Key words: Dental implantation, Rehabilitation, Osseointegration, Proton pump inhibitors, Risk factors

[paper submitted 2018. 7. 11 / revised 2018. 8. 5 / accepted 2018. 8. 8]

\section{Introduction}

Dental implants offer a predictable and effective solution for the treatment of total or partial edentulism with high survival rates; however, failures are still encountered ${ }^{1}$. The maintenance of a firm and long-term stable osseointegration is the primary goal of dental implant treatment. Implant failures can be classified as either early failures, which occur before the prosthesis is placed, and late failures, which are associated with functional loading following placement of the prosthe$\mathrm{sis}^{2}$. Early failures frequently occur as a result of a disruption during the initial phase leading to impaired bone-to-implant

\footnotetext{
Mehmet Ali Altay

Department of Oral and Maxillofacial Surgery, School of Dentistry, Akdeniz University, Dumlupinar Boulevard, Campus, Antalya 07058, Turkey

TEL: +90-532-4071945 FAX: +90-242-310-6967

E-mail:malialtay@hotmail.com

ORCID: https://orcid.org/0000-0001-8886-4783

(c) This is an open-access article distributed under the terms of the Creative Commons Attribution Non-Commercial License (http://creativecommons.org/ licenses/by-nc/4.0/), which permits unrestricted non-commercial use, distribution, and reproduction in any medium, provided the original work is properly cited. Copyright (C) 2019 The Korean Association of Oral and Maxillofacial Surgeons. All rights reserved.
}

contact $^{3}$, while the onset of late failures may be related to multiple variables such as overloading, oral microbiota, and parafunctional habits ${ }^{2}$. The incidence of early implant failure ranges from $0.7 \%$ to $2.0 \%{ }^{4}$. Despite the higher incidence of early implant failure ${ }^{5}$, many studies have focused more on the success of implant rehabilitation after prosthetic loading ${ }^{6,7}$, limiting the available knowledge of factors affecting initial bone apposition until abutment connection ${ }^{3}$.

By definition, osseointegration is a direct structural and functional connection between bone and the surface of a load-carrying implant ${ }^{8}$. The osseointegration process is influenced by various factors, which may be classified broadly as implant-related (e.g., material, macroscopic design, implant surface), surgical/prosthetic protocol-related (e.g., surgical technique, loading conditions, time) or patient-related (e.g., quantity/quality of bone, the host response) $)^{9}$. Based on the fact that early failures may occur even when appropriate conditions exist for both implant-related and surgical protocolrelated factors, specific patient-related factors that affect bone metabolism should also be taken into consideration when evaluating factors affecting osseointegration success ${ }^{2,10}$. 
Several factors affecting bone metabolism including age, sex, tobacco use, systemic diseases, radiotherapy, and some systemic medications have been suggested to play a role in early implant failures ${ }^{3,11}$. To date, the effects of pharmacological treatments on the osseointegration of dental implants have been subject to a number of studies, suggesting that the systemic intake of certain pharmacological agents may either improve or negatively affect the osseointegration process ${ }^{11-14}$. Among these, proton pump inhibitors (PPIs) have recently been suggested to impair the osseointegration of dental implants because of their adverse effects on bone metabo$\operatorname{lism}^{15-17}$.

PPIs have a wide range of applications in gastroenterology for the treatment of various disorders such as gastroesophageal reflux disease, peptic ulcers, dyspepsia, Helicobacter $p y$ lori infections, stress gastritis, and eosinophilic esophagitis ${ }^{18}$. PPIs irreversibly inhibit the proton pump in the acid-secreting parietal cells of the stomach and thereby suppress the gastric acidity ${ }^{19}$. Although the overall safety profile of PPIs is considered superior, there are recent studies that have indicated an association between PPI therapy and some adverse effects including enteric infections, community-acquired pneumonia, kidney disease, dementia, and bone fracture ${ }^{20}$.

PPIs may affect bone metabolism negatively by several mechanisms including the impairment of calcium absorption and hypergastrinemia-induced hyperparathyroidism ${ }^{21,22}$. Furthermore, it has been reported that PPIs may hinder osteoclast activity by acting on the vacuolar H+-ATPase (V-ATPase) of the osteoclasts ${ }^{23}$. Another effect of PPIs on bone metabolism is the decrease in osteoclastic differentiation mediated by osteoblastic cells ${ }^{23}$. Eventually, systemic intake of PPIs may provoke decreases in density, weight, cortical thickness, and mineral content as well as the biomechanical properties of the bone ${ }^{24}$.

Given the abovementioned effects, the question arises as to whether the intake of PPIs negatively affects osseointegration, due to the fact that the success of osseointegration is directly dependent on bone metabolism. However, the current literature offers only limited information on the effects of PPIs on dental implant osseointegration. Therefore, the present study sought to determine whether there is an association between systemic PPI intake and the early failure of dental implants.

\section{Materials and Methods}

A retrospective study including all patients who received implants between May 2012 and January 2017 at the Department of Oral and Maxillofacial Surgery at the Akdeniz University School of Dentistry (Antalya, Turkey) was designed in order to evaluate the association between PPI intake and osseointegration failure. This study was approved by the regional Ethical Review Board of Akdeniz University (09/08/2017; approval No. 555) and was conducted in full accordance with the protocols of the Declaration of Helsinki. All patients provided their written consent for surgical and prosthetic procedures as well as for clinical and radiological data acquisition.

Patients who were prescribed only PPIs for gastric disorders, presented with no other systemic conditions, and reported taking no other medications were included in this study. Patients with missing information for key variables were excluded. Additionally, those with a medical disorder known to negatively affect bone metabolism including hyperparathyroidism, hypothyroidism, hyperthyroidism, osteoporosis, osteomalacia, vitamin D deficiency, diabetes, Paget's disease, an oncologic condition, or a severe systemic condition (American Society of Anesthesiologists classification grades III or IV) were also excluded. Other exclusion criteria included pregnancy, smoking, alcoholism, and a history of radiotherapy applied to the head and neck region. In addition, patients who reported taking medications other than PPIs including immunosuppressive, antithrombotic, antihypertensive, antiepileptic, or psychiatric medications; corticosteroids; medications for asthma or high cholesterol levels; or bisphosphonates were also not included in this study.

All study participants received periodontal therapy-either phase I or phases I and II-prior to the placement of dental implants. By the time of implant placement, all patients were regular attendees of their screening sessions at the Department of Periodontology and were free of active periodontal disease or other oral infections. Patients who had active periodontal disease at the time of surgery and those who refused to undergo periodontal therapy prior to implant placement were not included in this study. All patients were provided with implants - specifically, solid-screw type implants with sand-blasted, acid-etched, or titanium plasma-sprayed surfaces - using the classic two-stage protocol under local anesthesia by two experienced surgeons (M.A.A. and A.S.).

PPI intake was used as the predictor variable for this study, where a PPI user was defined as a patient who reported taking any one of the verified types of PPI medications (i.e., omeprazole, pantoprazole, lansoprazole, dexlansoprazole, esomeprazole, rabeprazole, dexrabeprazole, or a combina- 
tion of these) perioperatively. The outcome variable was osseointegration failure, which was defined as a condition necessitating implant removal prior to prosthetic loading due to advanced peri-implant bone loss and implant mobility. Age ( $<30,30-60$, or $>60$ years), sex (female or male), and implant location (anterior, premolar, or posterior - maxilla or mandible) were other variables investigated in this study. Relevant data from the records of patients that met the inclusion criteria were collected in an SPSS file for further analysis.

Descriptive statistics were recorded as numbers, percentages, or means \pm standard deviations. The Mann-Whitney U test was utilized to compare differences between PPI users and nonusers for continuous variables, whereas Fisher's exact test was applied for categorical variables depending on the expected count of events in a $2 \times 2$ contingency table.

Patient-level and implant-level models were implemented to investigate the effects of PPI intake on osseointegration failure. To verify multicollinearity, a correlation matrix of predictor variables with a significant odds ratio ( $P$-value cutoff point of 0.5 ) was tested to determine if there was any correlation among the predictors. The level of statistical significance was set at $P<0.05$. All data were analyzed statistically using the IBM SPSS Statistics software program (ver. 22; IBM Corp., Armonk, NY, USA).

\section{Results}

Five hundred ninety-two patients with a total of 1,918 implants met the eligibility criteria. Fifteen of these implants failed before prosthetic loading. One thousand twenty-three implants were placed in 316 female patients (53.4\%) and 895 implants were placed in 276 male patients (46.6\%) with mean ages of $48.96 \pm 13.15$ years (range, $18-84$ years) and $50.65 \pm$

Table 1. Chi-square tests for proton pump inhibitor status at the patient level

\begin{tabular}{|c|c|c|c|c|}
\hline & Value & $\begin{array}{l}\text { Asymp } \\
\text { Sig. } \\
\text { (two- } \\
\text { sided) }\end{array}$ & $\begin{array}{l}\text { Exact } \\
\text { Sig. } \\
\text { (two- } \\
\text { sided) }\end{array}$ & $\begin{array}{l}\text { Exact } \\
\text { Sig. } \\
\text { (one- } \\
\text { sided) }\end{array}$ \\
\hline Pearson chi-square test & 4.387 & 0.036 & \multirow{6}{*}{0.094} & \multirow{6}{*}{0.094} \\
\hline Continuity correction $^{1}$ & 1.914 & 0.166 & & \\
\hline Likelihood ratio & 2.668 & 0.102 & & \\
\hline Fisher's exact test & & & & \\
\hline Linear-by-linear association & 4.380 & 0.036 & & \\
\hline No. of valid cases & 592 & & & \\
\hline
\end{tabular}

(Asymp: asymptotic, Sig.: significance)

${ }^{1}$ Computed only for a $2 \times 2$ table.

Mehmet Ali Altay et al: Proton pump inhibitor intake negatively affects the osseointegration of dental implants: a retrospective study. J Korean Assoc Oral Maxillofac Surg 2019
14.21 years (range, 17-87 years), respectively. The difference in the number of implants between the sexes was not statistically significant ( $P=0.761$; Mann-Whitney $U$ test $)$.

Almost half of the implants in this study were placed in the mandible (957 implants; 49.9\%), with the remaining implants were placed in the maxilla (961 implants; 50.1\%). PPI users received more implants in the maxilla than in the mandible, whereas nonusers received more implants in the mandible, and the difference between two groups in this regard was found to be statistically significant $(P=0.027$; Fisher's exact test). Slightly more than one-quarter of the implants were placed at an anterior region (506 implants; 26.4\%), whereas premolar and molar regions received 603 implants (31.4\%) and 809 implants (42.2\%), respectively. Both nonusers and PPI users received more implants in a posterior region, but no statistically significant difference was found with respect to implant location ( $P=0.056$; Fisher's exact test $)$.

PPI users and nonusers had mean follow-up periods of $29.02 \pm 17.90$ and $28.97 \pm 17.59$ months, respectively $(P=0.724$; Mann-Whitney U test). The mean number of implants per patient was calculated to be $3.24 \pm 2.66$, ranging from one to 14 implants.

PPI users consisted of 18 female and six male patients with a total of 69 implants. Two PPI users experienced implant failure, one of whom had three and the other of whom had one failed implant, respectively; thus, the failure rate was calculated as $8.3 \%$ (2/24 patients). Eleven patients within the nonusers group each experienced one implant failure, making for a failure rate of $1.9 \%(11 / 568$ patients). Although the difference between PPI users and nonusers failed to show any significance at the patient-level ( $P=0.094$; Fisher's exact test) (Table 1), the difference at the implant-level was found to be statistically significant ( $P=0.002$; Fisher's exact test).(Table 2 )

Table 2. Chi-square tests for proton pump inhibitor status at the implant level

\begin{tabular}{lcccc}
\hline & Value & $\begin{array}{c}\text { Asymp } \\
\text { Sig. } \\
\text { (two- } \\
\text { sided) }\end{array}$ & $\begin{array}{c}\text { Exact } \\
\text { Sig. } \\
\text { (two- } \\
\text { sided) }\end{array}$ & $\begin{array}{c}\text { Exact } \\
\text { Sig. } \\
\text { (one- } \\
\text { sided) }\end{array}$ \\
\hline $\begin{array}{lccc}\text { Pearson chi-square test } \\
\text { Continuity correction }^{1}\end{array}$ & 23.199 & 0.000 & 0.002 & 0.002 \\
Likelihood ratio $^{\text {Fisher's exact test }}$ & 16.979 & 0.000 & & \\
Linear-by-linear association $_{\text {No. of valid cases }}^{10.192}$ & 0.001 & 0.002 & 0.002 \\
& 23.187 & 0.000 & 0.002 & 0.002 \\
\hline
\end{tabular}

(Asymp: asymptotic, Sig.: significance)

${ }^{1}$ Computed only for a $2 \times 2$ table.

Mehmet Ali Altay et al: Proton pump inhibitor intake negatively affects the osseointegration of dental implants: a retrospective study. J Korean Assoc Oral Maxillofac Surg 2019 
Table 3. Risk estimate for proton pump inhibitor (PPI) users

\begin{tabular}{lccr}
\hline & \multirow{2}{*}{ Value } & \multicolumn{2}{c}{$95 \%$ confidence interval } \\
\cline { 3 - 4 } & & Lower & Upper \\
\hline Odds ratio for PPI (user/nonuser) & 4.603 & 0.962 & 22.032 \\
For cohort fail=yes & 4.303 & 1.009 & 18.348 \\
For cohort fail=no & 0.935 & 0.828 & 1.055 \\
No. of valid cases & 592 & & \\
\hline
\end{tabular}

Mehmet Ali Altay et al: Proton pump inhibitor intake negatively affects the osseointegration of dental implants: a retrospective study.J Korean Assoc Oral Maxillofac Surg 2019

The odds of implant failure for patients on PPI medications in comparison with nonusers were calculated to be 4.60 times greater. Furthermore, dental implants that were placed in patients using PPIs were found to be 4.30 times more likely to fail prior to loading.(Table 3 )

\section{Discussion}

PPIs are some of the most commonly prescribed drugs for the treatment of acid-related gastric diseases ${ }^{18}$. Despite being well-tolerated drugs overall, several adverse effects have been linked with the use of PPIs including nausea, headaches, gastrointestinal symptoms, nutritional deficiencies, osteoporotic fractures, chronic kidney disease, infection, and thrombocytopenia $^{25,26}$.

The adverse effects of PPIs on bone metabolism may be explained through several potential mechanisms. First, PPIinduced hypochlorhydria leads to the malabsorption of calcium in the small intestine ${ }^{27}$. The resulting decrease in the blood calcium levels disturbs bone formation and stimulates bone resorption through osteoblasts and osteoclasts, respectively, leading to a lower degree of bone mineral density ${ }^{27,28}$. Secondly, PPIs may promote decreased bone turnover via the prevention of the V-ATPase of osteoclasts similar to the way PPIs inhibit gastric $\mathrm{H}^{+} / \mathrm{K}^{+}$-ATPase, which has a direct negative effect on bone cells ${ }^{23}$. Lastly, the long-term use of PPIs may impede collagen cross-linking and weaken the bone structure by causing an increase in homocysteine concentration and parathyroid hormone levels ${ }^{28}$.

Several observational studies have previously demonstrated an association between PPI exposure and fragility fractures, most notably of the hips and vertebrae $e^{18,20-22}$. Furthermore, in 2010, the United States Food and Drug Administration issued an advisory notice highlighting the possible risk for fractures of the hip, wrist, and spine when PPIs are used at high doses (i.e., more than once daily) or for a long duration (i.e., greater than one year). Nevertheless, controversy remains in the current literature regarding clinically significant effects of PPIs on bone metabolism ${ }^{29}$.

Today, the association between PPI intake and bone metabolism-related disorders remains a subject of interest for researchers. However, only a small number of studies to date have thus far investigated the effects of PPIs on the osseointegration of dental implants. In an in vivo study on rat tibia, $\mathrm{Al}$ Subaie et al. ${ }^{15}$ found that systemically administered omeprazole, a commonly prescribed $\mathrm{PPI}^{30}$, decreased the overall number of osteoclasts in the healing bone and had a deleterious effect on bone healing and implant osseointegration. Recently, Wu et al. ${ }^{17}$ reported on the association between PPIs and the risk of failure for osseointegrated implants in their retrospective cohort study covering 1,773 dental implants in 799 patients (including 133 implants in 58 PPI users), where the failure rates for PPI users and nonusers were found to be $6.88 \%$ and $3.2 \%$, respectively. These authors concluded that PPI use was associated with an increased risk of failure for osseointegrated implants. Similarly, Chrcanovic et al. ${ }^{16}$ evaluated the results from 999 patients (3,559 implants) and reported a $12 \%$ overall failure rate for PPI users and one of $4.5 \%$ for nonusers. The difference between the two groups was statistically significant. They ultimately suggested that the intake of PPIs might present a higher risk of dental implant failure. The results of the current study are consistent with the findings reported by $\mathrm{Wu}$ et al. ${ }^{17}$ and Chrcanovic et al. ${ }^{16}$, which revealed a statistically significant difference in implant failure rates between PPI users and nonusers.

The present study has the distinction of being the first attempt to evaluate the success of osseointegration in the presence of PPI intake by excluding patients who experienced late failures. It is noteworthy that the overall survival and success of dental implants are dependent on several factors including oral hygiene and parafunctional habits. Therefore, we believe that a more objective evaluation of PPI intake as a predictor of osseointegration failure was achieved by excluding patients who lost implants after prosthetic loading. To the best of our knowledge, this is the first study to investigate the association between PPI use and the success of dental implant osseointegration.

Certain limitations of this study must be borne in mind when interpreting its findings. The lack of specific information characterizing the type, dose, and duration of PPI therapy, which might have influenced the outcome, represents a limitation due to the retrospective design of the study. Moreover, as is true with all observational studies, our results may also be biased by some residual confounding factors. However, its findings are gleaned from a large sample size 
and may provide clinicians with insight into how the success of osseointegration is affected in the presence of PPI intake. It might also serve as the basis for future studies on this topic.

\section{Conclusion}

The findings of this study suggest that PPI intake may be associated with an increased risk of early failure of dental implants. Future prospective cohort studies and randomized controlled clinical trials are needed to validate our outcomes and more thoroughly elucidate the effects of PPI intake on the osseointegration process of dental implants.

\section{ORCID}

Mehmet Ali Altay, https://orcid.org/0000-0001-8886-4783

Alper Sindel, https://orcid.org/0000-0001-8760-5958

Öznur Özalp, https://orcid.org/0000-0003-4350-1975

Nelli Yildırımyan, https://orcid.org/0000-0001-9860-5494

Burak Kocabalkan, https://orcid.org/0000-0002-7717-3765

\section{Authors' Contributions}

M.A.A. and A.S. designed the study. M.A.A. helped draft the manuscript. N.Y. and B.K. participated in data collection. M.A.A., Ö.Ö., and N.Y. wrote the manuscript. Ö.Ö. and N.Y. performed the statistical analyses. All authors read and approved the final version of the manuscript.

\section{Ethics Approval and Consent to Participate}

This study was approved by the regional Ethical Review Board of Akdeniz University (09/08/2017; approval No. 555) and was conducted in full accordance with the protocols of the Declaration of Helsinki.

\section{Conflict of Interest}

No potential conflict of interest relevant to this article was reported.

\section{References}

1. Mangano F, Mortellaro C, Mangano N, Mangano C. Is low serum vitamin $\mathrm{D}$ associated with early dental implant failure? A retrospective evaluation on 1625 implants placed in 822 patients. Mediators Inflamm 2016;2016:5319718.

2. Chrcanovic BR, Kisch J, Albrektsson T, Wennerberg A. Factors influencing early dental implant failures. J Dent Res 2016;95:9951002.

3. Olmedo-Gaya MV, Manzano-Moreno FJ, Cañaveral-Cavero E, de Dios Luna-del Castillo J, Vallecillo-Capilla M. Risk factors associated with early implant failure: a 5-year retrospective clinical study. J Prosthet Dent 2016;115:150-5.

4. Koldsland OC, Scheie AA, Aass AM. Prevalence of implant loss and the influence of associated factors. J Periodontol 2009;80:106975.

5. Jemt T, Olsson M, Franke Stenport V. Incidence of first implant failure: a retroprospective study of 27 years of implant operations at one specialist clinic. Clin Implant Dent Relat Res 2015;17 Suppl 2:e501-10.

6. Berglundh T, Persson L, Klinge B. A systematic review of the incidence of biological and technical complications in implant dentistry reported in prospective longitudinal studies of at least 5 years. J Clin Periodontol 2002;29 Suppl 3:197-212; discussion 232-3.

7. Charyeva O, Altynbekov K, Zhartybaev R, Sabdanaliev A. Longterm dental implant success and survival--a clinical study after an observation period up to 6 years. Swed Dent J 2012;36:1-6.

8. Albrektsson T, Brånemark PI, Hansson HA, Lindström J. Osseointegrated titanium implants. Requirements for ensuring a longlasting, direct bone-to-implant anchorage in man. Acta Orthop Scand 1981;52:155-70.

9. Trindade R, Albrektsson T, Wennerberg A. Current concepts for the biological basis of dental implants: foreign body equilibrium and osseointegration dynamics. Oral Maxillofac Surg Clin North Am 2015;27:175-83.

10. Mangano C, Mangano F, Shibli JA, Ricci M, Sammons RL, Figliuzzi M. Morse taper connection implants supporting "planned" maxillary and mandibular bar-retained overdentures: a 5-year prospective multicenter study. Clin Oral Implants Res 2011;22:111724.

11. Apostu D, Lucaciu O, Lucaciu GD, Crisan B, Crisan L, Baciut M, et al. Systemic drugs that influence titanium implant osseointegration. Drug Metab Rev 2017;49:92-104.

12. Kellesarian SV, Abduljabbar T, Vohra F, Malignaggi VR, Malmstrom H, Romanos GE, et al. Role of local alendronate delivery on the osseointegration of implants: a systematic review and metaanalysis. Int J Oral Maxillofac Surg 2017;46:912-21.

13. Wu X, Al-Abedalla K, Eimar H, Arekunnath Madathil S, AbiNader S, Daniel NG, et al. Antihypertensive medications and the survival rate of osseointegrated dental implants: a cohort study. Clin Implant Dent Relat Res 2016;18:1171-82.

14. Wu X, Al-Abedalla K, Rastikerdar E, Abi Nader S, Daniel NG, Nicolau B, et al. Selective serotonin reuptake inhibitors and the risk of osseointegrated implant failure: a cohort study. J Dent Res 2014;93:1054-61.

15. Al Subaie A, Emami E, Tamimi I, Laurenti M, Eimar H, Abdallah $\mathrm{MN}$, et al. Systemic administration of omeprazole interferes with bone healing and implant osseointegration: an in vivo study on rat tibiae. J Clin Periodontol 2016;43:193-203.

16. Chrcanovic BR, Kisch J, Albrektsson T, Wennerberg A. Intake of proton pump inhibitors is associated with an increased risk of dental implant failure. Int J Oral Maxillofac Implants 2017;32:1097102.

17. Wu X, Al-Abedalla K, Abi-Nader S, Daniel NG, Nicolau B, Tamimi F. Proton pump inhibitors and the risk of osseointegrated dental implant failure: a cohort study. Clin Implant Dent Relat Res 2017;19:222-32.

18. Leontiadis GI, Moayyedi P. Proton pump inhibitors and risk of bone fractures. Curr Treat Options Gastroenterol 2014;12:414-23.

19. Stedman CA, Barclay ML. Review article: comparison of the pharmacokinetics, acid suppression and efficacy of proton pump inhibitors. Aliment Pharmacol Ther 2000;14:963-78.

20. Freedberg DE, Kim LS, Yang YX. The risks and benefits of longterm use of proton pump inhibitors: expert review and best practice 
advice from the American Gastroenterological Association. Gastroenterology 2017;152:706-15.

21. Abraham NS. Proton pump inhibitors: potential adverse effects. Curr Opin Gastroenterol 2012;28:615-20.

22. Yang YX, Lewis JD, Epstein S, Metz DC. Long-term proton pump inhibitor therapy and risk of hip fracture. JAMA 2006;296:294753.

23. Costa-Rodrigues J, Reis S, Teixeira S, Lopes S, Fernandes MH. Dose-dependent inhibitory effects of proton pump inhibitors on human osteoclastic and osteoblastic cell activity. FEBS J 2013;280:5052-64.

24. Dobrowolski P, Tomaszewska E, Radzki RP, Bienko M, Wydrych $\mathrm{J}$, Zdybel A, et al. Can 2-oxoglutarate prevent changes in bone evoked by omeprazole? Nutrition 2013;29:556-61.

25. Devlin JW, Welage LS, Olsen KM. Proton pump inhibitor formulary considerations in the acutely ill. Part 2: clinical efficacy, safety, and economics. Ann Pharmacother 2005;39:1844-51.

26. Yu LY, Sun LN, Zhang XH, Li YQ, Yu L, Yuan ZQ, et al. A review of the novel application and potential adverse effects of proton pump inhibitors. Adv Ther 2017;34:1070-86.

27. Serfaty-Lacrosniere C, Wood RJ, Voytko D, Saltzman JR, Pedrosa
M, Sepe TE, et al. Hypochlorhydria from short-term omeprazole treatment does not inhibit intestinal absorption of calcium, phosphorus, magnesium or zinc from food in humans. J Am Coll Nutr 1995; 14:364-8.

28. Boyce BF. Stomaching calcium for bone health. Nat Med 2009;15:610-2.

29. Lau YT, Ahmed NN. Fracture risk and bone mineral density reduction associated with proton pump inhibitors. Pharmacotherapy 2012;32:67-79.

30. Gunasekaran TS, Hassall EG. Efficacy and safety of omeprazole for severe gastroesophageal reflux in children. J Pediatr $1993 ; 123: 148-54$.

How to cite this article: Altay MA, Sindel A, Özalp Ö, Yıldırımyan N, Kocabalkan B. Proton pump inhibitor intake negatively affects the osseointegration of dental implants: a retrospective study. J Korean Assoc Oral Maxillofac Surg 2019;45:135-40. https://doi.org/10.5125/jkaoms.2019.45.3.135 